\title{
EL 1, 2, 3 DE LA EXPERIMENTACIÓN CON ANIMALES DE LABORATORIO
}

\author{
Wilber Romero-Fernandez , , Zenia Batista-Castro, ${ }^{1, b}$, Marisel De Lucca ${ }^{1, c}$, Ana Ruano, ${ }^{2, d}$, \\ María García-Barceló ${ }^{1, b}$, Marta Rivera-Cervantes ${ }^{3, e}$, Julio García-Rodríguez $z^{4, f}$, Soledad Sánchez-Mateos ${ }^{1, g}$
}

\begin{abstract}
RESUMEN
El lento desarrollo científico experimentado en América Latina en las últimas décadas ha retrasado la incorporación de la experimentación con animales de laboratorio, sin embargo, esta realidad ha comenzado a cambiar. En la actualidad, se evidencia un extraordinario progreso científico que ha promovido la introducción e incremento del uso de animales de laboratorio como una importante herramienta para el avance de las ciencias biomédicas. A raíz de este auge, surge la necesidad de proporcionar a la comunidad científica la formación y directrices en todos los aspectos relacionados con la experimentación animal. Es responsabilidad de cada país legislar esta práctica para que no solo por razones bioéticas, sino también legales, se considere el derecho de los animales y con ello su bienestar. El siguiente manuscrito es el resultado de las comunicaciones presentadas en el Taller Internacional en Ciencias de Animales de Laboratorio celebrado en la Universidad Técnica de Ambato, Ecuador; contiene la actualidad en la ciencia de animales de laboratorio, haciendo énfasis en aspectos fundamentales, tales como: principales especies utilizadas y su biología, principios éticos y legales, diseño experimental y alternativas al uso de animales, todas ellas encaminadas a garantizar las buenas prácticas que deben caracterizar el quehacer científico. Sin duda, este documento será relevante tanto para aquellos investigadores que pretenden incorporar la experimentación animal a sus investigaciones, como para aquellos que, aun teniendo experiencia, pretendan actualizar sus conocimientos.
\end{abstract}

Palabras clave: Experimentación animal, Bioética, Alternativas al uso de animales, Bienestar del animal, Ratones transgénicos (fuente: DeCS BIREME).

\section{THE 1, 2, 3 OF LABORATORY ANIMAL EXPERIMENTATION}

\begin{abstract}
The slow scientific development in Latin America in recent decades has delayed the incorporation of laboratory animal experimentation; however, this situation has started to change. Today, extraordinary scientific progress is evident, which has promoted the introduction and increased use of laboratory animals as an important tool for the advancement of biomedical sciences. In the aftermath of this boom, the need to provide the scientific community with training and guidance in all aspects related to animal experimentation has arisen. It is the responsibility of each country to regulate this practice, for both bioethical and legal reasons, to ensure consideration of the animals' rights and welfare. The following manuscript is the result of papers presented at the International Workshop on Laboratory Animal Testing held at the Technical University of Ambato, Ecuador; it contains information regarding the current state of affairs in laboratory animal testing and emphasizes critical aspects such as main species used, ethical and legal principles, and experimental and alternative designs for animal use. These works aim to ensure good practices that should define scientific work. This document will be relevant to both researchers who aim to newly incorporate animal testing into their research and those who seek to update their knowledge.
\end{abstract}

Key words: Animal experimentation, Bioethics, Animal use alternatives, Animal welfare, Transgenic mice (source: MeSH NLM).

\section{INTRODUCCIÓN}

El uso de animales en experimentación ha ido en paralelo al desarrollo de la biomedicina. Se define como animal de laboratorio a todo aquel ser vivo no humano, vertebrado o invertebrado, usado para la experimentación y otros fines científicos ${ }^{(1,2)}$; su uso se basa, fundamentalmente, en la analogía fisiológica con la especie humana. Entre

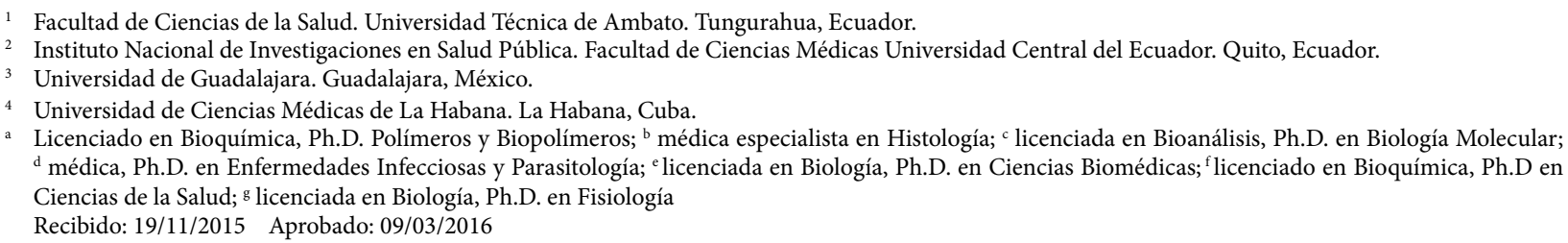

Citar como: Romero-Fernandez W, Batista-Castro Z, De Lucca M, Ruano A, García-Barceló M, Rivera-Cervantes M, García-Rodríguez J, Sánchez-Mateos S. El 1, 2, 3 de la experimentación con animales de laboratorio. Rev Peru Med Exp Salud Publica. 2016;33(2):288-99. doi: 10.17843/rpmesp.2016.332.2169 
los animales usados en investigación están los primates no humanos, prosimios, gatos, perros, reptiles, anfibios, ovejas, cerdos, cabras, peces, insectos y roedores, estos últimos son los de mayor uso $\mathrm{y}$, dentro de ellos, las ratas, ratones, conejos y cobayos ${ }^{(3)}$. A pesar de que muchos laboratorios aplican el principio de las tres erres (3R) planteado por Russell y Burch ${ }^{(4)}$, el número de animales utilizados en investigación se ha incrementado, realidad que es más acentuada en América Latina. No obstante, ya sea por razones éticas, por la necesidad de personal calificado, por los altos costos de producción y mantenimiento, o por los protocolos experimentales, cada vez se utilizan con mayor frecuencia alternativas a la experimentación animal ${ }^{(5)}$.

El punto de partida para el uso de animales en las investigaciones biomédicas es conocer los detalles biológicos (anatomía, fisiología y etología) y de mantenimiento (alojamiento, alimentación y manejo) de la especie. Detalles sobre la biología de los animales más utilizados en experimentación han sido descritos ampliamente en la literatura científica ${ }^{(6)}$.

\section{ASPECTOS BIOÉTICOS Y LEGALES}

La ciencia de animales de laboratorio surge para proporcionar a la comunidad científica la formación y directrices en todos los aspectos relacionados con la experimentación animal. Su marco ético plantea que los animales no deben ser sometidos a sufrimiento físico o psicológico y se regula por estrictas normas bioéticas ${ }^{(2,7)}$.

Cada país debe legislar el correcto cuidado y uso de los animales de experimentación y asegurar que gocen de las cinco libertades y las $3 R^{(8)}$. Para garantizar que la reglamentación sea la adecuada no solo se deben tomar en cuenta las normas de la legislación nacional, sino también las recomendaciones de organismos y asociaciones internacionales dedicadas a la ciencia de animales de laboratorio como: International Council of Laboratory Animal Science (ICLAS); Institute of Laboratory Animal Resources (ILAR); Universities Federation of Animal Welfare (UFAW); American Association for Laboratory Animal Science (AALAS); Interagency Research Animal Committee (IRAC); Animal Welfare Regulations (AWRs); Federación de Sociedades Sudamericanas de Ciencias en Animales de Laboratorio (FESSACAL) y Federation of European Laboratory Animal Science Associations (FELASA).

El uso de animales en experimentación es ético cuando no hay otra alternativa y su propósito está vinculado con la obtención de un bien mayor. Cada institución donde se realice esta práctica tiene que instaurar un comité institucional de cuidado y uso de animales de laboratorio (CICUAL), cuyoreglamentoestaráregidoporlalegislación nacional; si no la hubiese, se regirá por normativas internacionales. Para verificar que la investigación con animales se justifique por los beneficios que produce, el CICUAL debe revisar y aprobar todos los proyectos de investigación que impliquen experimentación animal. También, debe asegurar el correcto uso del bioterio, controlar el estado de salud de los animales y que su manipulación se realice de forma ética y humana. Además, ha de supervisar que el personal que trabaja con animales de laboratorio tenga la certificación que acredite su formación y experiencia para este fin ${ }^{(9)}$. Aunque existen diferentes criterios para constituir un CICUAL, se recomienda que esté formado, al menos, por un investigador con amplia experiencia en el trabajo con animales de experimentación, un veterinario y un miembro de la sociedad, este último preferiblemente de una asociación protectora de animales ${ }^{(10)}$.

Un posible criterio para evaluar las buenas prácticas bioéticas es el cumplimiento de las $3 R^{(4,5,11)}$, encaminado a: I). Reemplazar de forma parcial o total el uso de animales (5); II). Reducir el número de animales por experimento (12), y III). Refinar los procedimientos durante la cría, experimentación y eutanasia ${ }^{(13)}$.

\section{INFRAESTRUCTURA PARA LA EXPERIMENTACIÓN CON ANIMALES}

El lugar donde se alojan los animales de experimentación se denomina bioterio, animalario o estabulario, y se define como la instalación dedicada a la crianza, mantenimiento, cuidado y uso de los animales de laboratorio ${ }^{(14)}$.

El bioterio no solo debe cubrir las necesidades de los animales, sino también del personal que trabaja en él. Para ello, su infraestructura incluye diferentes áreas que se engloban en dos zonas: zona sucia (área administrativa, vestuario, almacenaje, eliminación de desechos e incineración) y zona limpia (área de recepción de animales, cuarentena, salas de alojamiento y crianza, laboratorios, quirófano y áreas de limpieza y esterilización).

Para evitar posibles contaminaciones, las zonas han de estar totalmente separadas por barreras físicas, y el flujo de trabajo se establece desde la zona limpia a la sucia. Además, en todas las áreas se tienen que seguir estrictas condiciones de higiene, limpieza, desinfección y estar perfectamente señalizadas ${ }^{(14)}$. Los protocolos de trabajo en el bioterio tienen que estar estandarizados, regulados según las normas éticas y legales, y supervisados por el CICUAL.

Es necesario que tanto el bioterio como su personal proporcionen al animal un perfecto estado de salud física y mental en sincronía con el ambiente, libre de hambre, sed 
y malnutrición; libre de miedo, ansiedad y angustia; libre de incomodidad física, frío, y calor; libre de dolor, lesión y enfermedad y libre para expresar su comportamiento natural ${ }^{(15)}$. Para garantizar estas cinco libertades, es imprescindible controlar una serie de parámetros, tanto del microambiente como del macroambiente. En cuanto al microambiente, se tendrán en cuenta las dimensiones de la jaula y la carga animal por jaula. Además, es importante proporcionar una dieta enriquecida y balanceada según el tipo de animal, agua fresca y libre de microorganismos y un encamado de calidad. Estos últimos elementos se deben cambiar con regularidad. Las jaulas y los biberones han de ser limpiados y desinfectados periódicamente. Es aconsejable, además, proporcionar un enriquecimiento en las jaulas con diferentes accesorios según la especie ${ }^{(1,2)}$. Se ha demostrado que un ambiente enriquecido disminuye el estrés y el miedo, facilita el manejo de los animales y favorece una rápida aclimatación (16).

Con respecto al macroambiente, es fundamental asegurar la higiene y desinfección de las salas de alojamiento, y controlar constantes ambientales como temperatura, humedad relativa, ventilación, iluminación, altas concentraciones de amoníaco y ruido. En la Tabla 1 se muestran las recomendaciones para el alojamiento de los roedores más utilizados en experimentación según la FELASA y la Convenio Europeo para la Protección de animales vertebrados usados para la experimentación y otros fines científicos ${ }^{(1)}$.

Dado el impacto del recurso humano en el bienestar animal, es inexcusable contar con un personal formado y experimentado. En este sentido, existen organizaciones internacionales que dictan cursos de capacitación periódicos para las diferentes categorías según el cargo y la labor por desarrollar. Según la FELASA, existen cuatro categorías: categoría $A$ (técnicos de laboratorio),categoría B (investigadores que realizan trabajos experimentales), categoría C (investigador principal de un proyecto) y categoría D (veterinario) ${ }^{(1,2)}$.

\section{LA SALUD DE LOS ANIMALES DEBE SER EVALUADA DE FORMA PERIÓDICA}

\begin{abstract}
El personal capacitado ha de evaluar el estado de salud de los animales de forma rutinaria, no solo por obligación legal y moral, sino también para garantizar resultados fiables y repetibles. Es necesario que esta evaluación sea más amplia y frecuente en procedimientos invasivos, quirúrgicos y a la llegada del animal al bioterio. En la Tabla 2 se muestra una propuesta de plantilla para la realización de esta evaluación.
\end{abstract}

Tabla 1. Recomendaciones para el alojamiento de los principales roedores utilizados en experimentación animal según la Federación de Asociaciones Europeas para la Ciencia en Animales de Laboratorio y la Convenio Europeo para la protección de animales vertebrados usados para la experimentación y otros fines científicos

\begin{tabular}{|c|c|c|}
\hline Bienestar animal* $^{*}$ & Ratones & Ratas \\
\hline \multirow{4}{*}{$\begin{array}{l}\text { Dimensiones de jaulas } \\
\text { (se puede incrementar durante } \\
\text { la cría) }\end{array}$} & Menos de $20 \mathrm{~g}$ de peso, $60 \mathrm{~cm}^{2}$ por animal & Menos de $200 \mathrm{~g}$ de peso, $200 \mathrm{~cm}^{2}$ por animal \\
\hline & $20-25 \mathrm{~g}$ de peso, $70 \mathrm{~cm}^{2}$ por animal & $200-400 \mathrm{~g}$ de peso, $300 \mathrm{~cm}^{2}$ por animal \\
\hline & $25-30 \mathrm{~g}$ de peso, $80 \mathrm{~cm}^{2}$ por animal & $400-600 \mathrm{~g}$ de peso, $450 \mathrm{~cm}^{2}$ por animal \\
\hline & Más de $30 \mathrm{~g}$ de peso, $100 \mathrm{~cm}^{2}$ por animal & Más de $600 \mathrm{~g}, 600 \mathrm{~cm}^{2}$ por animal \\
\hline Número de animales por jaula & $4-6$ & $2-4$ \\
\hline Cantidad alimento por día & $3-6 \mathrm{~g}$ & $10-20 \mathrm{~g}$ \\
\hline Ingesta de agua por día & 3-7 mL & $15-30 \mathrm{~mL}$ \\
\hline Temperatura & $20^{\circ} \mathrm{C}$ a $24^{\circ} \mathrm{C}$ & $20^{\circ} \mathrm{C}$ a $24^{\circ} \mathrm{C}$ \\
\hline Humedad & $45-65 \%$ & $45-65 \%$ \\
\hline Ruido & $<20 \mathrm{kHz}$ & $<20 \mathrm{kHz}$ \\
\hline Ciclos de luz/oscuridad & 12 horas de luz / 12 horas de oscuridad & 12 horas de luz / 12 horas de oscuridad \\
\hline Ventilación & $\begin{array}{l}\text { Ambiente controlado y purificado de } 15 \text { a } 18 \\
\text { recambios aire / hora. Animales en jaulas } \\
\text { ventiladas. Controlar niveles de } \mathrm{CO}_{2}\end{array}$ & $\begin{array}{l}\text { Ambiente controlado y purificado de } 15 \text { a } 18 \\
\text { recambios aire / hora. Animales en jaulas } \\
\text { ventiladas. Controlar niveles de } \mathrm{CO}_{2}\end{array}$ \\
\hline Olores & Controlar el nivel de amoníaco & Controlar el nivel de amoníaco \\
\hline Camaje & Pino blanco, sin olores y libre de patógenos & Pino blanco, sin olores y libre de patógenos \\
\hline Enriquecimiento de las jaulas & $\begin{array}{l}\text { Papel, cajas de cartón, bastidores para } \\
\text { escalar }\end{array}$ & $\begin{array}{l}\text { Tubos, bastidores para escalar, cajas de } \\
\text { cartón }\end{array}$ \\
\hline
\end{tabular}

*Estas recomendaciones pueden variar en función de la especie, la edad y el sexo del animal. Con ellas se garantizan las principales necesidades del animal, tales como la expresión de las posturas típicas, descanso, exploración, emparejamiento, construcción del nido, reproducción, forraje, alimentación, hidratación, necesidades fisiológicas, excavación, comportamiento social y estímulos ambientales 
Durante el mantenimiento de los animales, tiene especial interés la evaluación microbiológica y parasitológica (1). Los animales deben mantenerse libres de patógenos específicos (SPF) y de gérmenes (GF). Dentro de los patógenos con mayor riesgo para el bienestar animal se encuentra el virus Hanta y el virus de la coriomeningitis linfocítica. A esta lista se suman bacterias como Helicobacter spp., Salmonella spp., Mycobacterium tuberculosis, Campylobacter spp., Yersinia enterocolitica, Vibrio spp., Mycoplasma spp., Leptospira spp., Listeria spp. y Streptobacillus moniliformis (17), algunos hongos, tales como especies de los géneros Microsporum, Trichophyton y Epidermophyton que causan dermatofitosis (18) y varios parásitos, tales como Leishmania, Tripanosoma, Toxoplasma, Fasciola y tremátodos del género Schistosoma (17). Para un correcto monitoreo del estado microbiológico, se pueden consultar las recomendaciones de la FELASA ${ }^{(1)}$.

Es necesario evitar las enfermedades que se trasmiten tanto de los animales vertebrados al hombre como las que se trasmiten del hombre a estos animales, lo cual se logra manteniendo el buen estado de salud y trabajando en zonas de bioseguridad con las precauciones requeridas ${ }^{(19)}$.

Las alergias y reacciones respiratorias son comunes en el personal que trabaja con animales de laboratorio ${ }^{(20)}$. Entre los alérgenos más frecuentes se han descrito proteínas de la

Tabla 2. Propuesta de plantilla para evaluar el estado de los animales

\begin{tabular}{|c|c|c|}
\hline Observación & Evaluación & Comentario \\
\hline \multirow[t]{3}{*}{ Condiciones generales } & 0 & Comportamiento normal según la especie \\
\hline & 1 & Menos alerta, se asusta cuando se manipula, responde a estímulos umbrales e intensos \\
\hline & 4 & $\begin{array}{l}\text { Animal inmóvil, no responde a estímulos intensos, vocaliza, agresivo, miedo al contacto, pobre aseo, } \\
\text { dilatación de las pupilas, aumento de salivación y sudoración, orejas hacia atrás }\end{array}$ \\
\hline \multirow[t]{3}{*}{ Porfirina } & 0 & Ojos claros, limpios y húmedos \\
\hline & 1 & Escasa porfirina o secreción alrededor de los ojos y la nariz \\
\hline & 4 & Porfirina evidente y secreciones alrededor de los ojos y la nariz \\
\hline \multirow[t]{3}{*}{ Movimiento y postura } & 0 & Normal \\
\hline & 1 & Moderado descoordinación, postura ligeramente encorvada \\
\hline & 4 & $\begin{array}{l}\text { Marcada descoordinación, cabeza arqueada, postura encorvada, no apoyo en cuatro extremidades, } \\
\text { parálisis, contracciones reflejas de la musculatura esquelética }\end{array}$ \\
\hline \multirow[t]{3}{*}{ Piloerección } & 0 & Normal, limpio y ordenado \\
\hline & 1 & Moderado piloerección \\
\hline & 4 & Marcada piloerección, piel dañada, pelo sucio \\
\hline \multirow[t]{3}{*}{ Piel } & 0 & Piel cubierta totalmente por pelos \\
\hline & 1 & Pequeñas llagas o costras sin infección o signos de picor \\
\hline & 4 & Automutilación, mordedura a otros animales, signos de infección \\
\hline \multirow[t]{3}{*}{ Peso } & 0 & De 1-3 días, pérdida de peso de menos del $5 \%$ en comparación con el peso antes del experimento \\
\hline & 1 & De 1-3 días, pérdida de peso de menos del 5-10\% en comparación con el peso antes del experimento \\
\hline & 4 & $\begin{array}{l}\text { De } 1-3 \text { días, pérdida de peso de menos del } 10-20 \% \text { en comparación con el peso antes del } \\
\text { experimento }\end{array}$ \\
\hline \multirow[t]{3}{*}{ Apetito } & 0 & Apetito normal \\
\hline & 1 & $\begin{array}{l}\text { Moderado interés por la comida, disminución en el consumo de agua, hasta menos del } 25 \% \text { del } \\
\text { consumo de alimentos y agua }\end{array}$ \\
\hline & 4 & Desinterés por la comida, signos de deshidratación \\
\hline \multirow[t]{3}{*}{ Función } & 0 & Normal función intestinal y urinaria \\
\hline & 1 & Heces más sueltas que lo normal \\
\hline & 4 & $\begin{array}{l}\text { Estreñimiento, heces como pasas, diarreas prolongadas (más de } 3 \text { días) incremento o disminución } \\
\text { de la orina, olor fuerte en la orina }\end{array}$ \\
\hline \multirow[t]{3}{*}{ Respiración } & 0 & Normal \\
\hline & 1 & Intermitente respiración por la boca \\
\hline & 4 & $\begin{array}{l}\text { Respiración con la boca abierta, respiración abdominal o jadeo, ruidos jadeantes, respiración } \\
\text { laboriosa }\end{array}$ \\
\hline \multirow[t]{3}{*}{ Estado microbiológico } & 0 & Presencia de bacterias y hongos como flora normal \\
\hline & 1 & Infección bacteriana, fúngica parasitaria o viral que remite con tratamiento \\
\hline & 4 & Infección bacteriana, fúngica, parasitaria o viral que afecta el bienestar del animal \\
\hline
\end{tabular}

* Si la suma de los puntos de evaluación es igual o superior a 3 se recomienda consultar a un veterinario o proceder a la eutanasia de los animales 
orina, de la saliva y de la sangre, así como el pelo y la cama de los animales. Se recomienda un examen médico antes de comenzar a trabajar con animales de experimentación, así como exámenes periódicos ${ }^{(21)}$. El uso de jaulas ventiladas, el trabajo en cabinas de flujo laminar, el empleo de barreras biológicas y un buen sistema de circulación de aire reducen considerablemente la aparición de alergias. En la literatura científica se pueden consultar diferentes guías para la prevención de enfermedades ocupacionales relacionadas con el trabajo de animales de laboratorio ${ }^{(17,22)}$.

\section{LA SELECCIÓN GENÉTICA DE LOS ANIMALES REFINA LOS PROCEDIMIENTOS EXPERIMENTALES}

Los principales roedores utilizados en el laboratorio se pueden clasificar en cepas consanguíneas y colonias no consanguíneas. Las cepas consanguíneas se caracterizan por homogeneidad genética, isogenicidad, uniformidad fenotípica, larga estabilidad genética, identidad e individualidad de cepa, y son distribuidas internacionalmente con un fondo genético registrado. Las no consanguíneas maximizan la variación genética y minimizan la endogamia con una mayor variación fenotípica interindividual, lo que permite obtener animales con mejor fertilidad, salud y longevidad, útiles en análisis genéticos de rasgos complejos. Adicionalmente, existen otras líneas que pueden ser útiles dependiendo de los experimentos que se vayan a realizar, como son: híbridos de $\mathrm{F} 1$, líneas congénicas, coisogénicas, consómicas, y conplásticas, entre otras ${ }^{(23)}$.

Los ratones genéticamente modificados o transgénicos son un modelo animal cada vez más usado. Estos pueden modificarse, añadiendo genes exógenos para cambiar alguna característica, adicionando alguna funcionalidad (Knockin) o bloqueando la expresión de algún gen (Knockout). Existen diferentes tipos de ratones Knockin y Knockout cuya selección es un punto crítico en el diseño experimental (DE) y de ello dependen los resultados ${ }^{(24)}$. En la actualidad, existen bases de datos como Mouse Phenome Database en las que se sugieren las líneas a seleccionar de acuerdo al tipo de experimento.

Tanto los protocolos y métodos más empleados para la creación de un ratón transgénico, como su uso en la experimentación biomédica, pueden ser consultados en la literatura científica ${ }^{(25,26)}$.

En los últimos años, toda la información generada a partir de los estudios con ratones transgénicos está siendo recopilada por The International Mouse Phenotyping Consortium (27), cuya meta es generar un catálogo completo del fenotipo de los ratones mutantes con indicadores robustos para cada enfermedad y un protocolo estandarizado para eliminar las variables inherentes a los diferentes estudios. Este consorcio recopila información de diferentes grupos como The Sanger Institute Mouse Genetics Project (28) y The European Mouse Disease Clinic ${ }^{(29)}$. También ha incorporado líneas disponibles de ratones mutantes y células madre embrionarias mutadas provenientes del International Knockout Mouse Consortium (30). En su base de datos podemos realizar búsquedas actualizadas por fenotipo o por un gen específico. Además, brinda asesoría en la creación de transgénicos y dispone de líneas de roedores ya establecidas para la investigación científica.

\section{EL USO DE MODELOS ANIMALES Y UN CORRECTO DISEÑO EXPERIMENTAL REDUCEN EL NÚMERO DE ANIMALES EN UN EXPERIMENTO}

A pesar del alto costo y la dificultad que representa el desarrollo de un modelo animal, su uso nos permite estudiar la fisiopatología de una enfermedad inducida o espontánea, y el comportamiento animal. Dadas las posibles variaciones en las respuestas fisiológicas, es necesario tener un amplio conocimiento de la fisiología y anatomía comparada del modelo a emplear. Además, es aconsejable constatar la validez del modelo para el estudio. El ratón es el animal de laboratorio mejor caracterizado genéticamente. Si a ello sumamos su pequeño tamaño, fácil manejo, corto período gestacional, camadas grandes y rápida madurez sexual, todo ello lo convierte en una especie óptima para la creación de modelos ${ }^{(31)}$.

El desarrollo y aplicación de nuevos modelos animales para el estudio de la biología estructural y funcional está en constante crecimiento. En general, estos modelos tienen el propósito de comprender mecanismos biológicos (exploratorios), determinar una función biológica anormal (mecanísticos) o valorar y cuantificar el efecto de un tratamiento específico (predictivos). Estos pueden ser homólogos si los procesos que ocurren en el modelo son idénticos a los que ocurren en el humano; isomórficos si son semejantes, y parciales si se pueden emplear para el estudio de ciertos aspectos del proceso patológico o del tratamiento.

Los modelos animales son clasificados en modelos inducidos (experimentales), generados por modificación genética (transgénicos), espontáneos (genéticos), negativos y huérfanos ${ }^{(32,33)}$. Los tres primeros son los más frecuentes; sin embargo, hasta la fecha solo unos pocos modelos inducidos reproducen fielmente la etiología y fisiopatología de una enfermedad. Estos pueden lograrse experimentalmente por manipulación 
genética ${ }^{(34,35)}$ o quirúrgica, administración de dietas modificadas o de sustancias biológicamente activas ${ }^{(36)}$ y cambios de factores sociales o del entorno animal.

Un correcto DE no solo reduce el número de animales planteado en el principio de las $3 R$ s, sino que también garantiza el refinamiento de los procedimientos y la obtención de resultados fiables y repetibles. Son muchos los parámetros a fijar dentro de un DE, todos con impacto directo en el resultado experimental. En el DE es notable prestar especial atención al recurso humano y financiero. Una importante decisión es escoger la especie, sexo, edad y cepa por utilizar y los procedimientos experimentales se han de realizar de lo simple a lo complejo. Se tiene que estandarizar el inóculo, dosis, vía y frecuencia de la inoculación, definir el punto final de experimentación, analgesia y punto final experimental humanitario. Otro aspecto importante es determinar el tamaño de la muestra, basándose en procedimientos estadísticos sin superar el mínimo necesario para asegurar la fiabilidad de los resultados. Numerosa literatura científica describe los métodos estadísticos y ejemplos prácticos para calcular el número de animales en función de la investigación (37,38).

Las pruebas piloto cumplen un papel importante en el DE, ya que ayudan a definir y desarrollar la investigación (31). Es imprescindible revisar todos los resultados obtenidos durante la experimentación con el fin de validar o rectificar los procedimientos establecidos.

Para evitar errores sistemáticos o sesgos, los animales deben ser tratados de igual forma antes y durante el experimento, y el estudio se tiene que realizar de forma aleatoria y a ciegas; además, se han de incluir los correspondientes controles ${ }^{\left({ }^{11}\right)}$.

\section{CONSIDERAR CON ANTICIPACIÓN EL DOLORO SUFRIMIENTO SEGÚN LA AGRESIVIDAD DEL PROCEDIMIENTO EXPERIMENTAL}

El nivel de severidad de los procedimientos invasivos debe estar justificado en el proyecto de investigación y autorizado por el CICUAL. EI DE tiene que contar con un protocolo de refinamiento para garantizar la disminución del sufrimiento en los animales. También se han de establecer la analgesia y el punto final humanitario del experimento, los cuales pueden ser determinados por resultados previos, estudios pilotos y/o la monitorización del animal.

La fisiopatología y los tipos de dolor en animales de laboratorio han sido descritos en la literatura $(39,40)$. Con el fin de disminuir el umbral de activación de los nociceptores que incrementan notablemente el estrés en los animales es de suma importancia prestar atención a los procedimientos experimentales que causan sensibilización.

El dolor y el estrés tienden a alterar el balance homeostático del animal y provocan cambios endocrinos e inmunológicos, disminuyen el consumo de agua y alimentos, pueden causar complicaciones postoperatorias etc., todo ello, con un impacto negativo en los resultados experimentales. En la Tabla 3 se muestran algunos parámetros para evaluar el dolor y el malestar animal. En este sentido, Langford et al. 2010 han descrito un sistema de codificación conductual estandarizado basado en el dolor y la expresión facial (41).

El uso apropiado de anestésicos y analgésicos es un principio ético y científico ineludible que ayuda a evitar o minimizar el malestar, dolor y/o angustia de los animales. Existe numerosa literatura científica que aborda el manejo del dolor en los animales de experimentación (42,43), como las guías recomendadas por el CICUAL y la FELASA ${ }^{(44,45)}$.

La elección de fármaco(s), anestésico(s) y/o analgésico(s) debe responder a las necesidades clínicas sin comprometer la investigación. La vía de administración y la dosis dependerá de la especie, estado del animal, objetivo, duración del procedimiento, experiencia del investigador y medios disponibles (Tabla 4). Durante los procedimientos invasivos y a través de telemetría, es importante controlar la frecuencia cardiaca y respiratoria, la oxigenación, el color de las mucosas, la temperatura rectal, los fluidos y los reflejos, así como proporcionar protección ocular. Posteriormente, el animal se tiene que colocar en cama limpia, entorno tranquilo, incrementar la temperatura, suministrar alimentación especial, administrar analgesia y controlar postura, peso y balance de fluidos. Todo ello posibilita la recuperación del animal y una rápida cicatrización, además de reducir la alteración hormonal y la variabilidad experimental.

Cuando el sufrimiento sea mayor que el previsto y no sea posible mantener las condiciones de salud y bienestar, es necesario proceder a la eutanasia. Los métodos de eutanasia tienen que causar la pérdida inmediata de la conciencia, ser mínimamente invasivos y no dolorosos, y evitar en todo momento malestar o angustia al animal. Además, han de ser fáciles de realizar, reproducibles, irreversibles, realizados por personal experto y nunca en presencia de otros animales. La finalización del experimento se basa en el criterio humano, finalización del estudio o que los animales no sean aptos para la cría. Es obligatorio confirmar la muerte ya sea por el cese de la respiración o ritmo cardiaco, ausencia de reflejos, dilatación de las pupilas, o bien por desangramiento, evisceración, congelación o decapitación. Además, el animal se ha de mantener en congelación hasta su incineración. 
Tabla 3. Parámetros de comportamiento animal, fisiológicos y post mortem para evaluar el dolor y el malestar animal.

\begin{tabular}{|c|c|}
\hline Parámetros & Comentarios \\
\hline Comportamiento animal & $\begin{array}{l}\text { - Intranquilidad, miedo al contacto, agresividad } \\
\text { - Vocalización } \\
\text { - Protección de la parte dolorosa, lamido, mordisqueo o rascado del área afectada, } \\
\text { automutilación } \\
\text { - Animal encorvado, cambios en la forma de andar, tensión del abdomen, animal renuncia } \\
\text { a moverse } \\
\text { - Pérdida del apetito } \\
\text { - Pobre construcción del nido } \\
\text { - Pobre aseo }\end{array}$ \\
\hline Fisiológicos & $\begin{array}{ll}\text { - } & \text { Presencia de porfirina } \\
\text { - } & \text { Pérdida de peso, disminución del consumo de agua y alimentos } \\
\text { - } & \text { Piloerección } \\
\text { - } & \text { Cambios endocrinos e inmunológicos } \\
\text { - } & \text { Contracciones reflejas de la musculatura esquelética } \\
\text { - } & \text { Aumento de la presión arterial y la frecuencia cardíaca } \\
\text { - } & \text { Cambios en el patrón respiratorio } \\
\text { - } & \text { Aumento de la salivación y sudoración } \\
\text { - } & \text { Cambios en el brillo de los ojos y dilatación de las pupilas } \\
\text { - } & \text { Cambios en la expresión facial, posición de las orejas y del bigote } \\
\text { - } & \text { Disminución de la reproducción }\end{array}$ \\
\hline Post mortem & $\begin{array}{ll}\text { - } & \text { Depósito de grasa } \\
\text { - } & \text { Masa muscular } \\
\text { - } & \text { Tamaño de órganos linfoides y corteza adrenal } \\
\text { - } & \text { Balance de fluidos } \\
\text { - } & \text { Presencia de úlceras estomacales }\end{array}$ \\
\hline
\end{tabular}

Para aplicar los métodos de eutanasia hay que tener en cuenta la edad de los animales, la especie y el estado de salud. Una explicación detallada de cada uno de los métodos de eutanasia se puede obtener de la guía para la eutanasia de los animales de la Asociación Americana de Médicos Veterinarios ${ }^{(46)}$ y la FELASA ${ }^{(47)}$ (Tabla 5).

\section{EXISTEN ALTERNATIVAS A LA EXPERIMENTACIÓN CON ANIMALES DE LABORATORIO}

Entre las alternativas a la experimentación con animales de mayor percepción al dolor encontramos el uso de procariontes, protistas, hongos, invertebrados y vertebrados inferiores ${ }^{(48)}$. La alternativa más usada hasta la fecha son los cultivos celulares ${ }^{(49)}$. Los protocolos, tanto para los cultivos de células estables como para los cultivos primarios, están bien estandarizados y descritos en la literatura científica. Sin embargo, hay que tener en cuenta que una considerable cantidad de animales son sacrificados para obtener algunos de los suplementos necesarios para lograr el crecimiento celular, como el suero fetal bovino o equino.

Recientemente se ha reconstruido epidermis humana con características similares al tejido humano a partir de queratinocitos humanos cultivados en una matriz de colágeno. Esta epidermis es semejante en morfología, composición lipídica y marcadores bioquímicos. Actualmente, estos modelos son comercializados $y$, aunque su función de barrera comparada con la piel nativa está menos desarrollada, pueden ser una herramienta útil para ensayos de irritación, fototoxicidad y absorción percutánea, entre otras ${ }^{(50)}$.

Asimismo, se han utilizado organismos invertebrados tales como la Amphimedon queenslandica, Aplysia sp., Hydra, Drosophila melanogaster y Caenorhabditis elegans como modelos alternativos ${ }^{(48)}$. En un principio Drosophila melanogaster fue utilizado como modelo genético, sin embargo, en la actualidad se ha descrito como modelo para estudio del envejecimiento o enfermedades neurodegenerativas como la enfermedad de Parkinson (51) y la enfermedad de Alzheimer (52). Más del $70 \%$ de los genes humanos relacionados con enfermedades tiene su homólogo en el genoma de este insecto. Homophila y FlyBase son bases de datos que se han convertido en importantes herramientas a la hora de vincular genes de enfermedades genéticas humanas y la secuencia genómica de Drosophila melanogaster ${ }^{(53,54)}$.

El nemátodo Caenorhabditis elegans ha sido otra alternativa ampliamente utilizada ${ }^{(48)}$. Su transparencia a lo largo de su corta vida, biología, mantenimiento en 
Tabla 4. Principales anestésicos y analgésicos utilizados en ratas y ratones según las recomendaciones de la Asociación Americana para la Ciencia de Animales de Laboratorio y la Federación de Asociaciones Europeas para la Ciencia en Animales de Laboratorio

\begin{tabular}{|c|c|c|c|}
\hline Agente & $\begin{array}{c}\text { Ratas } \\
(\mathrm{mg} / \mathrm{kg}) / \mathrm{vía}\end{array}$ & $\begin{array}{l}\text { Ratones } \\
\text { dosis } \\
(\mathrm{mg} / \mathrm{kg}) / \mathrm{vía}\end{array}$ & Comentarios \\
\hline \multicolumn{4}{|l|}{ Analgesia* } \\
\hline Buprenorfina & $\begin{array}{l}0,025-0,075 \\
\text { SC, IP o IV }\end{array}$ & $\begin{array}{l}0,05-0,5 \\
\text { SC, IP o IV }\end{array}$ & $\begin{array}{l}\text { Analgesia preventiva y postoperatoria cada } 6-8 \mathrm{~h} \text {. Para procedimientos } \\
\text { mayores considerar analgesia multimodal con un AINE. Naloxona } \\
(0,01-0,1 \mathrm{mg} / \mathrm{kg} \text { IV o IM) para revertir efecto de la analgesia por opioides }\end{array}$ \\
\hline Butorfanol & $\begin{array}{l}0,05-2 \\
\text { SC, IP o IV }\end{array}$ & $\begin{array}{l}0,5-5 \\
\text { SC, IP o IV }\end{array}$ & $\begin{array}{l}\text { Analgesia preventiva y postoperatoria cada } 1-4 \mathrm{~h} \text {. Considerar analgesia } \\
\text { multimodal con un AINE. Naloxona }(0,01-0,1 \mathrm{mg} / \mathrm{kg} \text { IV o IM) para revertir } \\
\text { efecto de la analgesia por opioides }\end{array}$ \\
\hline Morfina & $\begin{array}{l}2-5 \\
\text { IP o SC }\end{array}$ & $\begin{array}{l}5-10 \\
\text { IP o SC }\end{array}$ & $\begin{array}{l}\text { Analgesia preventiva y postoperatoria en procedimientos muy invasivos. } \\
\text { Cada } 2-4 \mathrm{~h} \text {. Naloxona }(0,01-0,1 \mathrm{mg} / \mathrm{kg} \text { IV o IM) para revertir efecto de la } \\
\text { analgesia por opioides }\end{array}$ \\
\hline Carprofeno & $\begin{array}{l}4-5 \\
\text { SC }\end{array}$ & $\begin{array}{l}4-5 \\
\text { IP u O }\end{array}$ & $\begin{array}{l}\text { Analgesia preventiva y postoperatoria cada } 12-24 \mathrm{~h} \text {. Se puede utilizar en } \\
\text { combinación con buprenorfina }\end{array}$ \\
\hline Meloxican & $\begin{array}{l}0,3-1 \\
\mathrm{IP}, \mathrm{IM}, \mathrm{SC} \text { u } \mathrm{O}\end{array}$ & $\begin{array}{l}0,3-1 \\
I P \text { u O }\end{array}$ & $\begin{array}{l}\text { Analgesia preventiva y postoperatoria cada } 24 \mathrm{~h} \text {. Se puede utilizar en } \\
\text { combinación con buprenorfina }\end{array}$ \\
\hline Ketoprofeno & $\begin{array}{l}2-5 \\
\text { SC }\end{array}$ & $\begin{array}{l}2-5 \\
\text { SC }\end{array}$ & $\begin{array}{l}\text { Analgesia preventiva y postoperatoria cada } 12-24 \mathrm{~h} \text {. Se puede utilizar en } \\
\text { combinación con buprenorfina }\end{array}$ \\
\hline Ketorolaco & $\begin{array}{l}3-5 \\
\mathrm{O}\end{array}$ & $\begin{array}{l}5-7,5 \\
\text { SC u O }\end{array}$ & $\begin{array}{l}\text { Analgesia preventiva y postoperatoria cada } 12-24 \mathrm{~h} \text {. Se puede utilizar en } \\
\text { combinación con buprenorfina }\end{array}$ \\
\hline Flunixino Meglumina & $\begin{array}{l}1-2 \\
\text { SC o IM }\end{array}$ & $\begin{array}{l}1-2 \\
\text { SC }\end{array}$ & $\begin{array}{l}\text { Analgesia postoperatoria cada } 12 \text { - } 24 \text { h, no utilizar más de } 3 \text { días. Útil en } \\
\text { el tratamiento de la hipertermia }\end{array}$ \\
\hline $\begin{array}{l}\text { Lidocaína } \\
\text { (anestésico local) }\end{array}$ & $\begin{array}{l}\text { Diluir hasta } \\
0,5 \% \text { SC }\end{array}$ & $\begin{array}{l}\text { Diluir hasta } \\
0,5 \% \text { SC }\end{array}$ & $\begin{array}{l}\text { No exceder de } 7 \mathrm{mg} / \mathrm{kg} \text {. Usar localmente antes de hacer la incisión } \\
\text { quirúrgica. Inicio de analgesia rápida pero de poca duración }(<1 \mathrm{~h})\end{array}$ \\
\hline $\begin{array}{l}\text { Bupivacaina } \\
\text { (anestésico local) }\end{array}$ & $\begin{array}{l}\text { Diluir hasta } \\
0,25 \% \text { SC }\end{array}$ & $\begin{array}{l}\text { Diluir hasta } \\
0,25 \% \text { SC }\end{array}$ & $\begin{array}{l}\text { No exceder de } 8 \mathrm{mg} / \mathrm{kg} \text {. Usar localmente antes de hacer la incisión } \\
\text { quirúrgica. Inicio de la analgesia más lento que la lidocaína, pero con } \\
\text { más duración }(\sim 4-8 \mathrm{~h}) \text {. }\end{array}$ \\
\hline \multicolumn{4}{|l|}{ Anestesia* } \\
\hline $\begin{array}{l}\text { Isoflurano o } \\
\text { sevoflurano }\end{array}$ & $\begin{array}{l}\text { Hasta } 5-8 \% \\
\text { para inducción } \\
1-3 \% \text { para } \\
\text { mantenimiento }\end{array}$ & $\begin{array}{l}\text { Hasta } 5-8 \% \\
\text { para inducción } \\
1-3 \% \text { para } \\
\text { mantenimiento }\end{array}$ & Utilizar en jaula de inducción y con analgesia preventiva \\
\hline Dióxido de carbono & Más del 70\% & Más del 70\% & $\begin{array}{l}\text { Para procedimiento terminal como la recogida de sangre seguido de la } \\
\text { eutanasia }\end{array}$ \\
\hline Ketamina-Xilacina & $\begin{array}{l}40-80+5-10 \\
\mathrm{IP}\end{array}$ & $\begin{array}{l}50-100+10-15 \\
\text { IP }\end{array}$ & $\begin{array}{l}\text { Mayor dosis de ketamina que de xilacina se recomienda para } \\
\text { procedimientos pocos invasivos y para animales jóvenes, muy viejos } \\
\text { en estado crítico. Proporciona de } 30-45 \mathrm{~min} \text {. Parcialmente revertido con } \\
\text { Atipamezol ( } 1-2 \mathrm{mg} / \mathrm{kg} \text { en ratones y } 0,1-1 \text { en ratas, IP, IM, SC o IV) o } \\
\text { yohimbina ( } 1-2 \mathrm{mg} / \mathrm{kg} \text { IP o SC). Esta última más específica a xilacina }\end{array}$ \\
\hline $\begin{array}{l}\text { Ketamina-Xilacina- } \\
\text { Acepromacina }\end{array}$ & $\begin{array}{l}40-80+5-10+1 \\
I P\end{array}$ & $\begin{array}{l}50-75+10-15 \\
+2 \\
\mathrm{IP}\end{array}$ & $\begin{array}{l}\text { La acepromacina extiende el tiempo de anestesia para procedimientos } \\
\text { extensos (cóctel } 65: 13: 2 \mathrm{mg} / \mathrm{kg}) \text {. Proporciona de } 20-30 \mathrm{~min} \text {. Parcialmente } \\
\text { revertido con atipamezol (1-2 mg/kg en ratones y 0,1-1 en ratas, IP, IM, } \\
\text { SC o IV) o yohimbina ( } 1-2 \mathrm{mg} / \mathrm{kg} \text { IP o SC) }\end{array}$ \\
\hline $\begin{array}{l}\text { Ketamina- } \\
\text { Medetomidina }\end{array}$ & $\begin{array}{l}40-80+0,5-1 \\
\text { IP }\end{array}$ & $\begin{array}{l}50-75+0,5-1 \\
\text { IP }\end{array}$ & Parcialmente revertido con atipamezol \\
\hline $\begin{array}{l}\text { Ketamina- } \\
\text { Midazolam }\end{array}$ & $\begin{array}{l}75-100+4-5 \\
\text { IP }\end{array}$ & $\begin{array}{l}80-100+4-5 \\
I P\end{array}$ & Para inmovilización. Proporciona de 20-30 minutos. \\
\hline $\begin{array}{l}\text { Pentobarbital de } \\
\text { Sodio }\end{array}$ & $\begin{array}{l}30-50 \\
\text { IP }\end{array}$ & $\begin{array}{l}50-90 \\
\text { IP }\end{array}$ & $\begin{array}{l}\text { Recomendado para procedimientos terminales. Puede ser apropiado } \\
\text { para cirugía craneal. Considerar analgesia con opioides o AINE. Largo } \\
\text { período de recuperación. Mantener la temperatura de los animales }\end{array}$ \\
\hline Tribromoetanol & $\begin{array}{l}200-240 \\
\text { IP }\end{array}$ & $\begin{array}{l}200-240 \\
\text { IP }\end{array}$ & $\begin{array}{l}\text { Induce la anestesia rápidamente y proporciona una buena analgesia } \\
\text { quirúrgica durante aproximadamente } 1 \mathrm{~h} \text {. Apropiado para procedimientos } \\
\text { a corto plazo. Recomendado para procedimientos terminales. Usar } \\
\text { soluciones frescas }\end{array}$ \\
\hline Propofol & $\begin{array}{l}12-26 \\
\text { IV }^{-12}\end{array}$ & $20-30$ & $\begin{array}{l}\text { Administración intravenosa, utilidad limitada en ratones. Puede causar } \\
\text { depresión respiratoria. Proporciona de 5-7 min }\end{array}$ \\
\hline
\end{tabular}

*El investigador valorará incrementar la dosis en procedimientos que causen un mayor daño tisular o el uso de dosis repetidas para una mayor duración de la anestesia. Subcutáneo (SC), intraperitoneal (IP), intravenoso (IV), intramuscular (IM) y oral (O) 
Tabla 5. Principales métodos químicos y físicos utilizados en la eutanasia de animales de laboratorio. Adaptación de las recomendaciones de la Federación de Asociaciones Europeas para la Ciencia en Animales de Laboratorio y de la Asociación Americana de Médicos Veterinarios

\begin{tabular}{|c|c|c|c|c|c|c|c|}
\hline Agente & & $\mathbf{R}^{*}$ & $E^{*}$ & $\mathbf{F}^{*}$ & $\mathbf{S}^{*}$ & $A^{*}$ & Comentarios \\
\hline $\begin{array}{l}\text { Isoflurano, enflurano, } \\
\text { sevoflurano }\end{array}$ & haloetano, & +++ & +++ & ++ & + & +++ & $\begin{array}{l}\text { Animales }<7 \mathrm{~kg} \text {. Puede utilizarse como } \\
\text { anestésico antes de otro método de eutanasia. } \\
\text { Se pueden utilizar con o sin óxido de nitrógeno } \\
\left(\mathrm{N}_{2} \mathrm{O}\right) \text {, pero el } \mathrm{N}_{2} \mathrm{O} \text { no debe emplearse solo para } \\
\text { eutanasia }\end{array}$ \\
\hline Pentobarbital de sodio & & +++ & +++ & + & ++ & +++ & $\begin{array}{l}\text { Recomendable. Administración intravenosa. } \\
\text { Para eutanasia es } 3 \text { veces la dosis de anestesia }\end{array}$ \\
\hline Ketamina & & +++ & +++ & + & ++ & +++ & $\begin{array}{l}\text { Utilizado en combinación con un agonista de } \\
\text { los receptores a } 2 \text {-adrenérgicos como xilacina o } \\
\text { benzodiacepinas como el diazepam }\end{array}$ \\
\hline Tribromoetanol & & ++ & ++ & + & ++ & ++ & Necesita ser administrado en dosis correctas \\
\hline Conmoción cerebral & & ++ & ++ & + & ++ & - & Menos de $1 \mathrm{~kg}$, nunca con martillo o hacha \\
\hline Dislocación cervical & & ++ & ++ & + & & & $\begin{array}{l}\text { Método utilizado en neonatos, ratones y ratas } \\
\text { de menos de } 200 \mathrm{~g} \text {. Realizado por personal } \\
\text { entrenado o en animales anestesiados }\end{array}$ \\
\hline T-61 & & ++ & ++ & - & + & ++ & $\begin{array}{l}\text { Realizado por personal entrenado y por } \\
\text { administración intravenosa muy lenta }\end{array}$ \\
\hline Dióxido de carbono $\left(\mathrm{CO}_{2}\right)$ & & + & ++ & ++ & ++ & ++ & $\begin{array}{l}\text { Al parecer no hay ventajas en el suministro de } \\
\text { oxígeno conjuntamente con } \mathrm{CO}_{2}\end{array}$ \\
\hline Irradiación con microonda & & ++ & ++ & - & ++ & + & $\begin{array}{l}\text { Método utilizado cuando se necesita fijación de } \\
\text { metabolitos en el cerebro }\end{array}$ \\
\hline Decapitación & & + & + & + & ++ & - & $\begin{array}{l}\text { Realizado por personal entrenado y en animales } \\
\text { anestesiados }\end{array}$ \\
\hline Rápida congelación & & - & + & ++ & ++ & + & Neonatos $<$ de $4 \mathrm{~kg}$, previa anestesia \\
\hline
\end{tabular}

${ }^{*} \mathrm{R}$ : rápida en perdida de la conciencia; E: eficacia; F: facilidad; S: seguridad del operario; A: nivel de anestesia del método. +++, alto; ++, moderado alto; +, normal y -, bajo. Métodos de eutanasia no aceptados: Administración de nitrógeno o argón, administración de cloruro de potasio, de agentes bloqueadores neuromusculares, opioides, uretano, cloralosa, ciclopropano, hipotermia, asfixia y monóxido de carbono

el laboratorio y facilidad de realizar el silenciamiento génico lo convierte en un candidato para estudios de diabetes, envejecimiento, cáncer ${ }^{(55)}$, obesidad ${ }^{(56)}$ y enfermedades neurodegenerativas ${ }^{(57)}$.

Además, algunos hongos de los géneros Neurospora crassa, Schizosaccharomyces pombe, Aspergillus nidulans, Saccharomyces cerevisiae y Cunnunghamella elegans pueden utilizarse como modelos alternativos (48). Cunnunghamella elegans ha sido utilizado para el estudio del metabolismo de drogas, tales como antidepresivos tricíclicos, relajantes musculares, antipsicóticos, antihistamínicos y anticolinérgicos ${ }^{(58)}$. También son varias las ventajas que hacen de Saccharomyces cerevisiae uno de los microorganismos eucariotas más versátiles en los estudios en biomedicina ${ }^{(48)}$.

En los últimos años el pez cebra (Danio rerio) se ha convertido en un excelente modelo alternativo a la experimentación con animales vertebrados de mayor percepción al dolor. Este ha sido utilizado en el estudio de enfermedades tan diversas como las neurodegenerativas ${ }^{(59)}$, las causadas por hongos y bacterias ${ }^{(60,61)}$ y las oncológicas ${ }^{(62,63)}$. El hecho de compartir más del $80 \%$ del genoma con los humanos, su capacidad reproductiva y el rápido desarrollo son algunas de las ventajas que hacen atractivo este modelo en investigación ${ }^{(64)}$. A pesar de ser animales resistentes, estos son susceptibles a parásitos intracelulares como microsporidios de Pseudoloma neurophilia, Oodinium y algunas especies de Mycobacterium ${ }^{(65)}$. Los detalles sobre su biología, genética e información del organismo como modelo animal pueden consultarse en el Centro Internacional Para el Trabajo con Pez Cebra (Zebrafish International Resource Center, ZIRC) ${ }^{(66)}$ y la Red de Información sobre el Pez Cebra (Zebrafish Information Network, ZFIN) ${ }^{(67)}$.

Finalmente, el uso de la bioinformática, no solo por sus resultados, sino también por la alta velocidad de procesamiento y costos, ha revolucionado la experimentación animal. Actualmente, a través de programas informáticos es posible diseñar nuevos medicamentos, simular posibles efectos biológicos y tóxicos e incluso predecir el sitio de unión de un fármaco a su receptor ${ }^{(48)}$. Entre las herramientas más utilizadas se encuentran el diseño de drogas asistido por ordenador (Computer Aided Drug Design, CADD) ${ }^{(68)}$, la predicción 
de la actividad biológica basada en la estructura química (Structure Activity Relationship, SARs) y las propiedades físico-químicas del fármaco (Quantitative Structure Activity Relationship, QSAR) ${ }^{(48)}$.

\section{CONCLUSIONES}

Los roedores como ratas y ratones siguen siendo las especies más utilizadas en investigación biomédica. Su uso en experimentación solo es ético cuando no hay otra alternativa y su propósito está vinculado con la obtención de un bien mayor. Es inexcusable que los proyectos de investigación que incluyan el uso de animales no sean revisados, aprobados y supervisados por el CICUAL. Además, es fundamental que desde su nacimiento hasta su muerte, a estos animales se les garantice su bienestar durante los procedimientos de cría, mantenimiento y experimentación. Especial atención se debe prestar al diseño experimental y la selección del modelo animal, aspectos que permiten refinar los protocolos de trabajo.
Finalmente, a pesar de su creciente uso, es importante resaltar que las alternativas a la experimentación con animales de laboratorio no solo deben ser utilizadas sino también desarrolladas.

Contribución de autoría: WR-F, ZB-C, MDL, MG-B, SS-M, $A R, M R C$ y JCR participaron en la concepción y diseño del artículo así como en la redacción y revisión crítica. WR-F y SS-M realizaron la aprobación de su versión final.

Fuentes de financiamiento: este trabajo ha sido realizado como parte de los proyectos de investigación financiados por la Dirección de Investigación y Desarrollo de la Universidad Técnica de Ambato para WR-F con resolución 1243-CUP-2014, SS-M con resolución 1370-CU-P-2014 y MDL con resolución 0805-CU-P-2015, así como el proyecto titulado "Implementación de una plataforma científica para el desarrollo de líneas de investigación en el área de la toxicología, inmunología, microbiología y biología molecular y celular" para WR-F y SS-M, y del Programa Becas Prometeo de la Secretaría Nacional de Educación Superior, Ciencia, Tecnología e Innovación del Ecuador para WR-F, MDL y SS-M.

Conflictos de interés: los autores declaran no tener conflicto de interés.

\section{REFERENCIAS BIBLIOGRÁFICAS}

1. Guillen J. FELASA guidelines and recommendations. J Am Assoc Lab Anim Sci. 2012;51(3):311-21.

2. Smith JA, van den Broek FA, Martorell JC, Hackbarth H, Ruksenas O, Zeller W, et al. Principles and practice in ethical review of animal experiments across Europe: summary of the report of a FELASA working group on ethical evaluation of animal experiments. Lab Anim. 2007;41(2):143-60. doi: $10.1258 / 002367707780378212$

3. National Research Council (US) and Institute of Medicine (US) Committee on Use of Laboratory Animals in Biomedical and Behavioral Research. Use of Laboratory Animals in Biomedical and Behavioral Research. Washinton, D.C.: National Academy Press (US); 1998. Diponible en: http://www.ncbi. nlm.nih.gov/books/NBK218262/

4. Russell WMS, Bursh RL. The Principles of Humane Experimental Technique. London: Methuen, London; 1959.

5. Balls M. Replacement of animal procedures: alternatives in research, education and testing. Lab Anim. 1994;28(3):193-211. doi: $10.1258 / 002367794780681714$

6. Martín-Zúñiga J, Orellana-Muriana JM, Tur Marí J. Ciencia y tecnología del animal de laboratorio.vol.I/II:2 (Textos Universitarios Ciencias Sanitarias). Madrid: Universidad de Alcalá, Servicio de Publicciones; 2009.
7. National Research Council. Institute of Laboratory Animal Resources. Guide for the Care and Use of Laboratory Animals. $8^{\text {th }}$ ed. Washinton, D.C.: National Academy Press; 2011.

8. Fentener van Vlissingen JM, Borrens M, Girod A, Lelovas P, Morrison F, Torres YS. The reporting of clinical signs in laboratory animals: FELASA Working Group Report. Lab Anim. 2015 Oct;49(4):267-83. doi: $10.1177 / 0023677215584249$.

9. ccac.ca [internet]. Ottawa: Canadian Council on Animal Care in Science; 2006 [citando el 28 de abril de 2016]. disponible en: http://www.ccac.ca/ en_/standards/guidelines

10. National Research Council Committee for the Update of the Guide for the Care and Use of Laboratory Animals. Guide for the Care and Use of Laboratory Animals. Press WNA, editor2011.

11. Flecknell P. Replacement, reduction and refinement. ALTEX. 2002;19(2):73-8. PubMed PMID: 12098013.

12. Kimber I, Pichowski JS, Betts CJ, Cumberbatch M, Basketter DA, Dearman RJ. Alternative approaches to the identification and characterization of chemical allergens. Toxicol In Vitro. 2001;15(4-5):307-12.

13. Hendriksen CF. Replacement, reduction and refinement alternatives to animal use in vaccine potency measurement.
Expert Review Vaccines. 2009;8(3):31322. doi: $10.1586 / 14760584.8 .3 .313$.

14. Kollmus H, Post R, Brielmeier M, Fernandez J, Fuchs H, McKerlie C, et al. Structural and functional concepts in current mouse phenotyping and archiving facilities. J Am Assoc Lab Anim Sci. 2012;51(4):418-35.

15. Graham ML, Prescott MJ. The multifactorial role of the $3 \mathrm{Rs}$ in shifting the harm-benefit analysis in animal models of disease. Eur J Pharmacol. 2015;759:19-29. doi: 10.1016/j. ejphar.2015.03.040.

16. Van de Weerd HA, Aarsen EL, Mulder A, Kruitwagen CL, Hendriksen CF, Baumans V. Effects of environmental enrichment for mice: variation in experimental results. J Appl Anim welf Sci. 2002;5(2):87-109.

17. Coelho AC, Garcia Diez J. Biological Risks and Laboratory-Acquired Infections: A Reality That Cannot be Ignored in Health Biotechnology. Front Bioeng Biotechnol. 2015;3:56. doi: 10.3389/fbioe.2015.00056.

18. Connole MD, Yamaguchi H, Elad D, Hasegawa A, Segal E, Torres-Rodriguez JM. Natural pathogens of laboratory animals and their effects on research. Med Mycol. 2000;38 Suppl 1:59-65.

19. Hankenson FC, Johnston NA, Weigler BJ, Di Giacomo RF.Zoonoses of occupational health importance in contemporary 
laboratory animal research. Comp Med. 2003;53(6):579-601.

20. Westall L, Graham IR, Bussell J. A riskbased approach to reducing exposure of staff to laboratory animal allergens. Lab Anim (NY). 2015;44(1):32-8. doi: 10.1038/laban.603.

21. Palmberg L, Sundblad BM, Lindberg A, Kupczyk M, Sahlander K, Larsson $\mathrm{K}$. Long term effect and allergic sensitization in newly employed workers in laboratory animal facilities. Respir Med. 2015. 109(9):1164-73. doi: 10.1016/j.rmed.2015.06.007.

22. Miller JM, Astles R, Baszler T, Chapin $\mathrm{K}$, Carey R, Garcia L, et al. Guidelines for safe work practices in human and animal medical diagnostic laboratories. Recommendations of a CDC-convened, Biosafety Blue Ribbon Panel. MMWR Suppl. 2012;61(1):1-102.

23. Benavides FJ, and Guénet, J-L. Manual de genética de roedores de laboratorio: Principios básicos y aplicaciones. Madrid: Universidad de Alcalá y SECL; 2003.

24. Miller RL. Transgenic mice: beyond the knowckout. Am J Physiol Renal Physiol. 2011;300(2):F291-300. doi: 10.1152/ ajprenal.00082.2010.

25. Giraldo P, Montoliu L. Size matters: use of YACs, BACs and PACs in transgenic animals. Transgenic Res. 2001;10(2):83-103.

26. Cho A, Haruyama N, Kulkarni AB. Generation of transgenic mice. Curr Protoc Cell Biol. 2009; Chapter 19:Unit 19.11. doi: 10.1002/0471143030. cb1911s42.

27. Koscielny G, Yaikhom G, Iyer V, Meehan TF, Morgan H, Atienza-Herrero J, et al. The International Mouse Phenotyping Consortium Web Portal, a unified point of access for knockout mice and related phenotyping data. Nucleic Acids Res. 2014;42(Database issue):D802-9. doi: $10.1093 / \mathrm{nar} / \mathrm{gkt} 977$.

28. White JK, Gerdin AK, Karp NA, Ryder E, Buljan M, Bussell JN, et al. Genomewide generation and systematic phenotyping of knockout mice reveals new roles for many genes. Cell. 2013;154(2):452-64. doi: 10.1016/j. cell.2013.06.022.

29. Ayadi A, Birling MC, Bottomley J, Bussell J, Fuchs H, Fray M, et al. Mouse large-scale phenotyping initiatives: overview of the European Mouse Disease Clinic (EUMODIC) and of the Wellcome Trust Sanger Institute Mouse Genetics Project. Mamm
Genome. 2012;23(9-10):600-10. doi: 10.1007/s00335-012-9418-y.

30. Bradley A, Anastassiadis K, Ayadi A, Battey JF, Bell C, Birling MC, et al. The mammalian gene function resource: the International Knockout Mouse Consortium. Mamm Genome. 2012;23(9-10):580-6. doi: 10.1007/ s00335-012-9422-2.

31. Navarro-Hernández J, Ramírez ROA, Villagrán C. Manual de procedimientos recomendables para la investigación con animales. México D.C.: SAMSARA; 2012.

32. Davidson MK, Lindsey JR, Davis JK. Requirements and selection of an animal model. Isr J Med sci. 1987;23(6):551-5.

33. Coppola A, Moshe SL. Animal models. Handb Clin Neurol. 2012;107:63-98. doi: $\quad 10.1016 /$ B978-0-444-528988.00004-5.

34. Kawaharada K, Kawamata M, Ochiya T. Rat embryonic stem cells create new era in development of genetically manipulated rat models. World J Stem cells. 2015 26;7(7):1054-63. doi: 10.4252/wjsc.v7.i7.1054.

35. Lee H. Genetically engineered mouse models for drug development and preclinical trials. Biomol Ther (Seoul). 2014;22(4):267-74. doi: 10.4062/ biomolther.2014.074.

36. Teste IS, Tamos YM, Cruz YR, Cernada AM, Rodriguez JC, Martinez NS, et al. Dose effect evaluation and therapeutic window of the neuro-EPO nasal application for the treatment of the focal ischemia model in the Mongolian gerbil. TheScientificWorldJournal. 2012;2012:607498. $10.1100 / 2012 / 607498$

37. Faul F, Erdfelder E, Lang AG, Buchner A. G*Power 3: a flexible statistical power analysis program for the social, behavioral, and biomedical sciences. Behav Res Methods. 2007;39(2):175-91.

38. Bate T, Clark R. The Design and Statistical Analysis of Animal Experiments. Cambridge and New York: Cambridge University Press; 2014.

39. Gauriau C, Bernard JF. Pain pathways and parabrachial circuits in the rat. Exp Physiol. 2002;87(2):251-8.

40. Sui P, Watanabe $\mathrm{H}$, Ossipov $\mathrm{MH}$, Bakalkin G, Artemenko K, Bergquist J. Proteomics of neuropathic pain: proteins and signaling pathways affected in a rat model. J Proteome Res. 2014;13(9):3957-65. doi: 10.1021/ pr500241q.
41. Langford DJ, Bailey AL, Chanda ML, Clarke SE, Drummond TE, Echols S, et al. Coding of facial expressions of pain in the laboratory mouse. Nat methods. 2010;7(6):447-9. 10.1038/nmeth.1455.

42. Flecknell P, Waterman-Pearson A, editores. Pain Management in Animals. $1^{\text {st }}$ ed. New York, NY: W.B. Saunders; 2000.

43. Berry SH. Analgesia in the Perioperative Period. Vet Clin North Am Small Anim Pract. 2015;45(5):1013-27. doi: 10.1016/j.cvsm.2015.04.007.

44. Pain and distress in laboratory rodents and lagomorphs. Report of the Federation of European Laboratory Animal Science Associations (FELASA) Working Group on Pain and Distress accepted by the FELASA Board of Management November 1992. Lab Anim. 1994;28(2):97-112.

45. National Research Council (US) Committee on Recognition and Alleviation of Pain in Laboratory Animals. Recognition and Alleviation of Pain in Laboratory Animals. Washington, D.C.: National Academy Press; 2009.

46. al. LSe. AVMA Guidelines for the Euthanasia of Animals. Edition 2013. Illinois; American Veterinary Medical Association; 2013.

47. Close B, Banister K, Baumans V, Bernoth EM, Bromage N, Bunyan J, et al. Recommendations for euthanasia of experimental animals: Part 2. DGXT of the European Commission. Lab Anim. 1997;31(1):1-32.

48. Doke SK, Dhawale SC. Alternatives to animal testing: A review. Saudi Pharm J. 2015;23(3):223-9. doi: 10.1016/j. jsps.2013.11.002.

49. Heinonen T. Better science with human cell-based organ and tissue models. Altern Lab Anim. 2015;43(1):29-38.

50. NetzlaffF, Lehr CM, Wertz PW, Schaefer UF. The human epidermis models EpiSkin, SkinEthic and EpiDerm: an evaluation of morphology and their suitability for testing phototoxicity, irritancy, corrosivity, and substance transport. Eur J Pharm Biopharm. 2005;60(2):167-78. doi:10.1016/j. ejpb.2005.03.004

51. West RJ, Furmston R, Williams CA, Elliott CJ. Neurophysiology of Drosophila models of Parkinson's disease. Parkinsons Dis. 2015;2015:381281. doi: $10.1155 / 2015 / 381281$. 
52. Prussing K, Voigt A, Schulz JB. Drosophila melanogaster as a model organism for Alzheimer's disease. Mol Neurodegener. 2013;8:35. doi: 10.1186/1750-1326-8-35.

53. Chien S, Reiter LT, Bier E, Gribskov M. Homophila: human disease gene cognates in Drosophila. Nucleic Acids Res. 2002;30(1):149-51.

54. Drysdale R, FlyBase Consortium. FlyBase: a database for the Drosophila research community. Methods Mol Biol. 2008;420:45-59. doi: 10.1007/978-159745-583-1_3.

55. Kirienko NV, Mani K, Fay DS. Cancer models in Caenorhabditis elegans. Dev Dyn. 2010;239(5):1413-48. doi: 10.1002/dvdy.22247.

56. Tserevelakis GJ, Megalou EV, Filippidis G, Petanidou B, Fotakis C, Tavernarakis N. Label-free imaging of lipid depositions in C. elegans using thirdharmonic generation microscopy. PLoS One. 2014;9(1):e84431. doi: 10.1371/ journal.pone.0084431

57. Alexander AG, Marfil V, Li C. Use of Caenorhabditis elegans as a model to study Alzheimer's disease and other neurodegenerative diseases. Front Genet. 2014;5:279. doi: 10.3389/ fgene.2014.00279.

58. Moody JD, Zhang D, Heinze TM, Cerniglia CE. Transformation of amoxapine by Cunninghamella elegans. Appl Environ Microbiol. 2000;66(8):3646-9.

59. Xi Y, Noble S, Ekker M. Modeling neurodegeneration in zebrafish. Curr Neurol Neurosci Rep. 2011;11(3):27482. doi: 10.1007/s11910-011-0182-2.

60. van Leeuwen LM, van der Sar AM, Bitter W. Animal models of tuberculosis: zebrafish. Cold Spring Harb Perspect Med. 2015;5(3):a018580. doi: 10.1101/ cshperspect.a018580.

61. Torraca V, Masud S, Spaink HP, Meijer AH. Macrophage-pathogen interactions in infectious diseases: new therapeutic insights from the zebrafish host model. Dis Model Mech. 2014;7(7):785-97. doi: 10.1242/ dmm.015594.

62. Barriuso J, Nagaraju R, Hurlstone A. Zebrafish: a new companion for translational research in oncology. Clin Cancer Res. 2015;21(5):969-75. doi: 10.1158/1078-0432.CCR-14-2921.

63. Blackburn JS, Langenau DM. Zebrafish as a model to assess cancer heterogeneity, progression and relapse. Dis Model Mech. 2014 Jul;7(7):755-62. doi: 10.1242/dmm.015842.

64. Sipes NS, Padilla S, Knudsen TB. Zebrafish: as an integrative model for twenty-first century toxicity testing. Birth Defects Res C Embryo Today.
2011;93(3):256-67. doi: 10.1002/ bdrc.20214.

65. Murray KN, Dreska M, Nasiadka A, Rinne M, Matthews JL, Carmichael $\mathrm{C}$, et al. Transmission, diagnosis, and recommendations for control of Pseudoloma neurophilia infections in laboratory zebrafish (Danio rerio) facilities. Comp Med. 2011;61(4):322-9.

66. Nasiadka A, Clark MD. Zebrafish breeding in the laboratory environment. ILAR J. 2012;53(2):161-8.

67. Sprague J, Clements D, Conlin T, Edwards P, Frazer K, Schaper K, et al. The Zebrafish Information Network (ZFIN): the zebrafish model organism database. Nucleic Acids Res. 2003;31(1):241-3.

68. Qiao LS, Zhang YL. [Application of CADD on multi-target drug R\&D in natural products]. Zhongguo Zhong Yao Za Zhi. 2014;39(11):1951-5.

Correspondencia: Wilber Romero Fernández. Dirección: C/ Salvador entre México y Colombia, Ingahurco. Facultad de Ciencias de la Salud

Universidad Técnica de Ambato. Ambato 180150, Ecuador

Teléfono: 59333730268 ext. 5239

Correo electrónico:wromfdez@uta.edu.ec

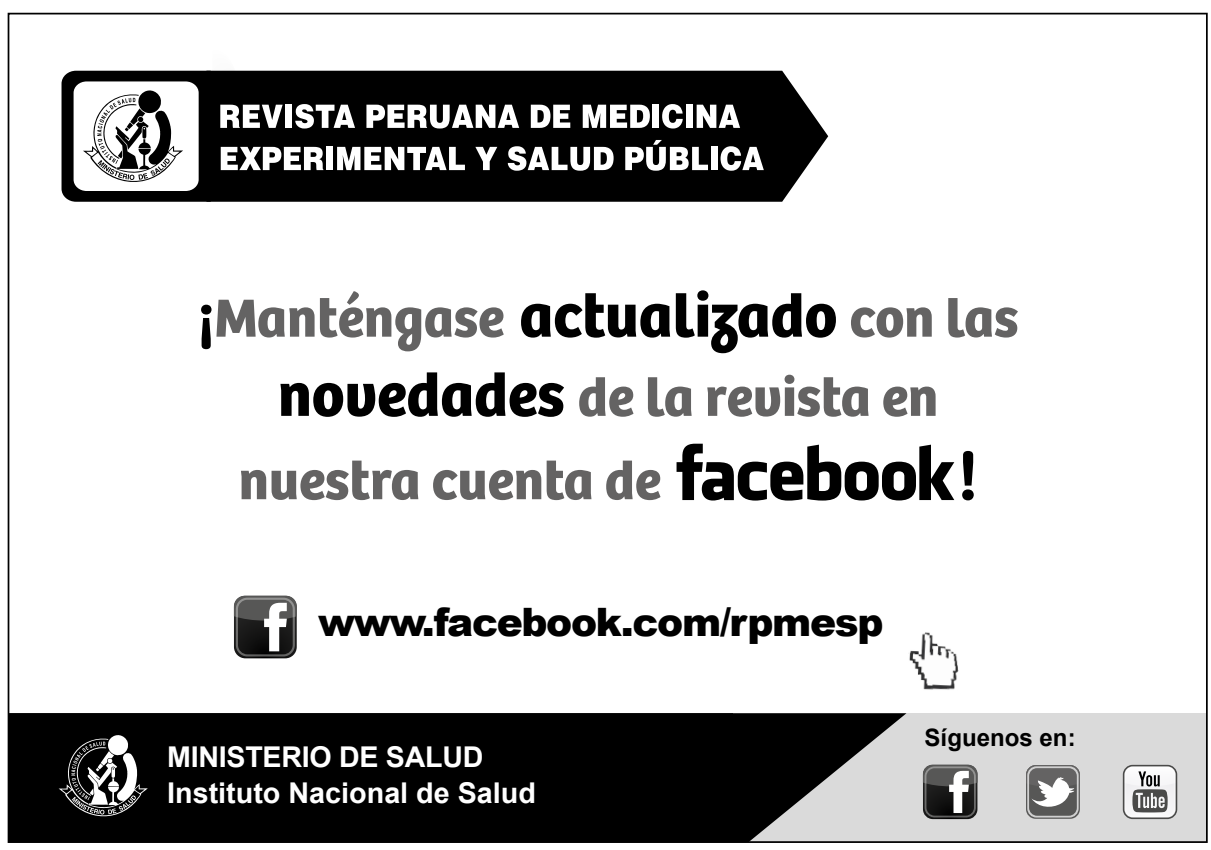

